\title{
Mode Analysis of Different Step Index Optical Fibers at 1064nm for High Power Fiber Laser and Amplifier
}

\author{
Arpan Dutta \\ Dept. of Physics, University of Burdwan, Burdwan, West Bengal, India
}

\begin{abstract}
Fiber lasers having high power and high beam quality are popular for various industrial, biomedical, defense and scientific applications. Rare earth doped active fibers are used as gain medium in fiber laser system. The core region of these fibers is doped with different rare earth elements like erbium (Er), ytterbium (Yb) and thulium (Tm). In this paper, observation of mode profiles and calculation of modal power of different step index fibers has been done with the help of COMSOL and MATLAB. The analysis was done at $1064 \mathrm{~nm}$ wavelength as these fibers are Yb-doped active fiber having an operation region near 1 micron for laser and amplifier applications. As we move to larger core diameter active fiber to achieve high power, the higher order modes starts carrying more power. To analyze this, fractional power of different transverse modes in the fiber both core and cladding region was calculated. Fibers were modeled in COMSOL and analyzed using MATLAB.
\end{abstract}

\section{Keywords}

Fiber Laser, Effective Refractive Index, Evanescent Field.

\section{Introduction}

Optical fibers can be used as active gain medium for building fiber lasers. The core regions of these fibers are doped with different rare earth elements such as erbium (Er), ytterbium (Yb), thulium (Tm) which provides gain at different wavelength regions. These fibers are called active fibers. Single mode fibers provide diffraction limited output but can't support high power due to their small core area. High power handling capability in fiber lasers can be achieved by using large mode area (LMA) fibers [1]. But due to large core, these fibers become multimoded. Multimode fibers can support high power but provide poor beam quality.

In the earlier stage of fiber laser technology, the first fiber lasers could deliver only a few mille watts of output power. Evolution of high power fiber laser took place after the demonstration of the concept of clad pumping technique using double clad LMA fibers where pump signal is propagated through clad and signal is propagated through core [2]. The average output power of Continuous Wave (CW) diffraction limited fiber lasers has been increased chronologically. In 1990s it was more than $100 \mathrm{~W}$ and in 2009 it reached at 10kW. Pulsed fiber lasers also developed rapidly. Pulsed lasers provide more than $100 \mathrm{~W}$ average power in 2005 and $1 \mathrm{~kW}$ in 2009 [3].

\section{Mode Theory of Step Index Fiber}

Modes in optical fiber are transverse electric field distributions propagated along the fiber axis. Waveguide has only a finite number of guided propagation modes. These modes have a unique effective refractive index $\left(n_{\text {eff }}\right)$ which should be greater than clad refractive index $\left(n_{2}\right)$ and less than core refractive index $\left(n_{1}\right)$ i.e. $\left(n_{2}<n_{\text {eff }}<n_{1}\right)$. Normalized propagation constant which is also unique for a particular mode is directly related with effective refractive index by this formula

$$
b=\frac{n_{e f f}^{2}-n_{2}^{2}}{N A^{2}}
$$

$$
N A=\sqrt{\left(n_{1}^{2}-n_{2}^{2}\right)}
$$

Where $\mathrm{NA}=$ numerical aperture of the fiber. $\mathrm{V}$ number is a dimensionless number and fixed for a fiber for a particular operating wavelength. It is used to define the mode propagation characteristics of a fiber. It is mathematically expressed as

$$
V=\frac{2 \pi}{\lambda} \times N A \times a
$$

Where $\mathrm{a}=$ fiber core radius and $\lambda=$ operating wavelength. For $\mathrm{V}<2.405$ fibers are always single mode i.e. It can support only one guided mode propagation on that optical wavelength. For $\mathrm{V}>2.405$, fibers are always multimode i.e. It can support more than one (multiple) guided mode propagation on that optical wavelength.

Any step index fiber (SIF) is characterized by the following refractive index (R.I.) distribution

$$
\begin{aligned}
\mathrm{n}(\mathrm{r}) & =\mathrm{n}_{1} \text { for } 0<\mathrm{r}<\mathrm{a}(\text { core }) \\
& =\mathrm{n}_{2} \text { for } \mathrm{r}>\mathrm{a}(\text { clad })
\end{aligned}
$$

If relative R.I. difference $\Delta=\frac{n_{1}-n_{2}}{n_{1}}<<1$, fibers are called weakly guiding [4]. Most practical fibers are weakly guiding as they have very small difference between core and clad R.I (approximately 0.01). If this difference is increased (strongly guided), NA of the fiber is increased. Hence the V number of the fiber crossed the single mode condition and becomes multimoded. As single mode operation is always preferred for better beam quality, weakly guiding approximation is considered for most practical fibers. In weakly guiding approximation, the modes are assumed to be nearly transverse with arbitrary state of polarization. Two independent sets of modes are assumed $-\mathrm{x}$ polarized and $\mathrm{y}$ polarized. They have same propagation constant. These modes are called Linearly Polarized (LP) modes [5].

The assumption of the continuity of modal field at core - clad interface gives the equation for studying the modes [5]. The equation is

$$
U \frac{J_{l+1}(U)}{J_{l}(U)}=W \frac{K_{l+1}(W)}{K_{l}(W)}
$$

$\mathrm{U}$ and $\mathrm{W}$ can be expressed in terms of normalized propagation constant (b) and $\mathrm{V}$ such that

$$
W=V \sqrt{b}
$$$$
U=V \sqrt{1-b}
$$

Using equation (1.2) in equation (1.1) we obtained For $1=0$ (fundamental mode)

$$
V(1-b)^{1 / 2} \frac{J_{1}\left[V(1-b)^{1 / 2}\right]}{J_{0}\left[V(1-b)^{1 / 2}\right]}=V b^{1 / 2} \frac{K_{1}\left[V b^{1 / 2}\right]}{K_{0}\left[V b^{1 / 2}\right]}
$$

For $1 \geq 1$ (higher order modes)

$$
V(1-b)^{1 / 2} \frac{J_{l-1}\left[V(1-b)^{1 / 2}\right]}{J_{l}\left[V(1-b)^{1 / 2}\right]}=-V b^{1 / 2} \frac{K_{l-1}\left[V b^{1 / 2}\right]}{K_{l}\left[V b^{1 / 2}\right]}
$$


The solutions of the transcendental equations (1.3) and (1.4) give us the dependence of $\mathrm{b}$ on $\mathrm{V}$. For a given value of $l$ there will be a finite number of solutions and the $\mathrm{m}^{\text {th }}$ solution $(\mathrm{m}=1,2,3 \ldots)$ is referred as the $\mathrm{LP}_{\mathrm{Im}}$ mode [5].

Modal power carried in the core is an important parameter of a fiber optic waveguide. The power in the core and clad of a fiber is mathematically given by

$$
\begin{aligned}
& P_{\text {core }}=C \pi a^{2}\left[1-\frac{J_{l-1}(U) J_{l+1}(U)}{J_{l}^{2}(U)}\right] \\
& P_{\text {clad }}=C \pi a^{2}\left[\frac{K_{l-1}(W) K_{l+1}(W)}{K_{l}^{2}(W)}-1\right]
\end{aligned}
$$

Where $\mathrm{C}=$ constant.

The fractional power propagating in the core is given by

$$
\eta=\frac{P_{\text {core }}}{P_{\text {total }}}=\left[\frac{W^{2}}{V^{2}}+\frac{U^{2}}{V^{2}} \frac{K_{l}^{2}(W)}{K_{l-1}(W) K_{l+1}(W)}\right]
$$

\section{Simulation Results}

Simulations are done for different SIF to plot the mode profiles and to calculate the modal power of various modes. Schematic design of different SIF are done in COMSOL using the known waveguide parameters of the fibers and COMSOL calculate the effective refractive indexes for all possible modes in the fiber using Finite Element Method (FEM). These effective refractive index values are the governing parameter of the analysis because normalized propagation constants (b) of each mode are calculated from these effective R.I values. As effective R.I. is unique for a particular mode, it gives b value for a specific mode. Fibers have unique $\mathrm{V}$ number for a particular operating wavelength. Using the $\mathrm{V}$ and $\mathrm{b}$ value, $\mathrm{U}$ and $\mathrm{W}$ values are calculated for a specific mode using equation (1.2). Thus with known values of $U$ and $\mathrm{W}$, calculation of the modal power contains in various modes is done using equation (1.5) and (1.6). Fractional power present in core is calculated using equation (1.7). The SIFs are analyzed at $1064 \mathrm{~nm}$ because fiber lasers designed in laboratory are $\mathrm{Yb}$ doped and operated at $1 \mu \mathrm{m}$. Waveguide parameters of different SIF are given in Table 1. Each fiber is modeled in COMSOL and analyzed in MATLAB.

Table 1: Waveguide Parameters of Different SIFs

\begin{tabular}{|c|c|c|c|c|}
\hline Fiber name & Core radius $(\mu \mathrm{m})$ & Core R.I. & NA & V number \\
\hline HI1060 & 2.65 & 1.4652 & .14 & 2.1908 \\
\hline RE22 & 6.25 & 1.4619 & .1 & 3.6908 \\
\hline RE64 & 12.5 & 1.4619 & .1 & 7.3816 \\
\hline
\end{tabular}

In this analysis, three fibers are chosen with different core diameter. HI1060 is standard single clad passive fiber and RE22 and RE64 are in-house made $\mathrm{Yb}$ doped double clad active fibers used in fiber laser and amplifier applications. HI1060 is single moded but RE22 and RE64 are multimoded at 1064nm. First, calculations of the values of effective R.I., b, U and $\mathrm{W}$ are done for various modes present in these fibers. Using these values, the percentage contribution by each mode in the total power present in a fiber is calculated.

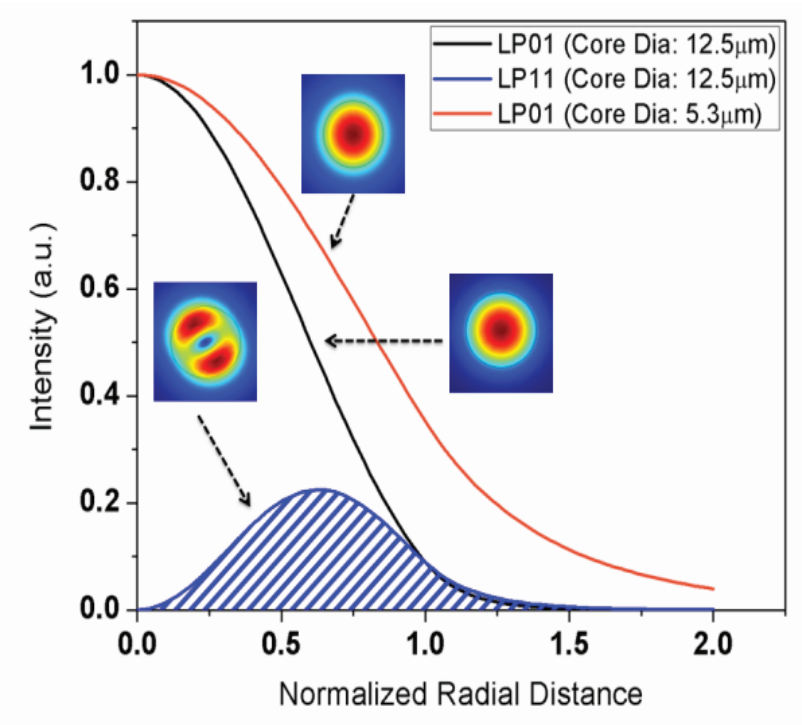

Fig. 1: Intensity profiles of various modes of RE22 and HI1060

In fig. 1, the intensity profiles of various modes of RE22 and HI1060 are plotted with respect to radial distance from fiber axis (normalized by core radius). The core-clad interface is implied by the value 1 in $\mathrm{x}$ axis. The COMSOL generated mode profiles are illustrated with corresponding intensity profiles. In HI1060, only fundamental mode is present but in RE22 a higher order mode is also present along with fundamental mode. The clad power or evanescent field is higher in HI1060 than that of RE22 as it is weakly guided. Due to strong guidance, RE22 has more power confined in the core. But its beam quality is poor than that of HI1060 due to presence of higher order mode (the blue shaded region). We can also conclude that the overlaps of higher order modes with the active ions (which are doped in the core uniformly) are less than that of fundamental mode.

Table 2: Power Contribution of Various Modes of HI1060

\begin{tabular}{|c|c|c|}
\hline Mode & Core power/ total power & $\%$ of total power in core \\
\hline LP01 & 0.7878 & $78.78 \%$ \\
\hline
\end{tabular}

In HI1060 only fundamental mode contributes power in the core. From table 2 we can say that $78.78 \%$ of total power of fiber is present in the core and rest $21.22 \%$ of total power of fiber is present in the clad as evanescent field.

Table 3: Power contribution of various modes of RE22

\begin{tabular}{|c|c|c|}
\hline Mode & Core power/ total power & $\%$ of total power in core \\
\hline LP01 & 0.6630 & $66.30 \%$ \\
\hline LP11 & 0.2413 & $24.13 \%$ \\
\hline
\end{tabular}

From Table 3 we can say that in RE22, 90.43\% of total power of fiber is present in the core in which $66.3 \%$ is contribution of LP01 mode and $24.13 \%$ is contribution of LP11 mode. Rest $9.57 \%$ of total power of fiber is present in the clad as an evanescent field. From table 4 it is clearly understood that in RE64, 96.45\% of total power of fiber is present in the core and rest $3.55 \%$ of total power of fiber is present in the clad as evanescent field. 


\section{HII060}

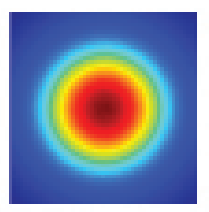

\section{LP01}

\section{RE22}

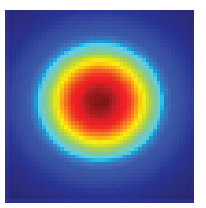

LP01

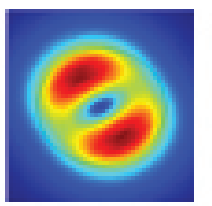

LP11

\section{RE64}

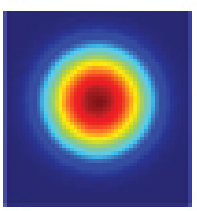

LP01

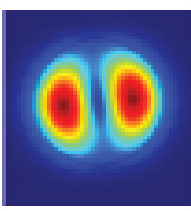

LP1 1

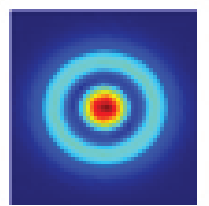

LP02

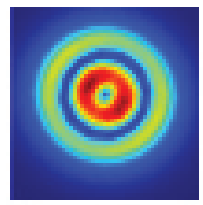

LP12

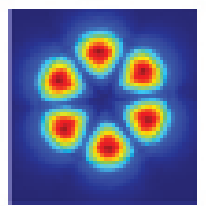

LP31

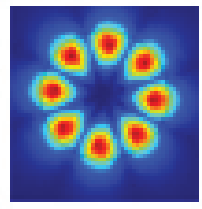

LP41

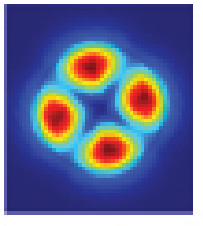

LP2 1

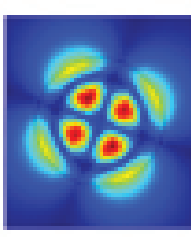

LP22

Fig. 3: COMSOL Generated Mode Profiles for Different SIF

Table 4: Power contribution of various modes of RE64

\begin{tabular}{|c|c|c|}
\hline Mode & Core power/ total power & $\%$ of total power in core \\
\hline LP01 & 0.5680 & $56.80 \%$ \\
\hline LP11 & 0.1402 & $14.02 \%$ \\
\hline LP21 & 0.0692 & $6.92 \%$ \\
\hline LP02 & 0.0587 & $5.87 \%$ \\
\hline LP31 & 0.0420 & $4.20 \%$ \\
\hline LP12 & 0.0347 & $3.47 \%$ \\
\hline LP41 & 0.0286 & $2.86 \%$ \\
\hline LP22 & 0.0231 & $2.31 \%$ \\
\hline
\end{tabular}


increment in core radius results increment in $\mathrm{V}$ number and hence total number of modes is increased. Due to increment in the number of higher order modes, power contribution of fundamental mode (in total power of the fiber present in the core) is decreased which is shown in figure 4. More a fiber is multimoded, less power contributed by the fundamental mode. More the fibers are multimoded, more power of the fiber present in the core and less power of the fiber present in the clad (evanescent field). Actually strongly guided fiber will have less evanescent field while being multimoded but single mode fiber or weakly guiding fiber will have more evanescent field [4]. Thus multimode fibers can provide high power than single mode. More core area of a fiber, more the power confined in the core. This conclusion implies that to increase the power handling capability of a fiber, it's necessary to increase the core area of the fiber. This leads to the importance of LMA fibers in high power fiber laser applications.

\section{V . Acknowledgement}

I would like to express my heartiest gratitude to Dr. Mrinmay Pal, Senior Scientist, Fiber Optics and Photonics Division (FOPD), CSIR-Central Glass and Ceramic Research Institute (CGCRI), for his valuable guidance and co-operation to complete this work. I am also thankful to the Director of the CSIR-CGCRI for giving me the opportunity to use the CGCRI laboratory and scientific software for my work.

\section{References}

[1] Fabian Stutzki, Florian Jansen, Hans-Jurgen Otto, Cesar Jauregui, Jens Limpert, Andreas Tunnermann, "Designing advanced very-large-mode-area fibers for power scaling of fiber laser systems", Vol. 1, No. 4 / October 2014 / Optica.

[2] D. J. Richardson, J. Nilsson, W. A. Clarkson, "High power fiber lasers: current status and future perspectives", Vol. 27, No. 11/November 2010/J. Opt. Soc. Am. B

[3] Cesar Jauregui, Jens Limpert, Andreas Tünnermann, "High Power Fiber laser", Nature photonics, Vol. 7, November 2013, pp. 861-867.

[4] Gloge D, "Weakly Guiding Fibers”, Appl. Opt. 1971, Vol. 10, No. 10.

[5] Ghatak, Thyagarajan,"Introduction to Fiber Optics", Cambridge university press, South Asia edition, 1999. 